\title{
Significance of glutathione S-transferases M1, T1 and P1 polymorphisms in Swedish melanoma patients
}

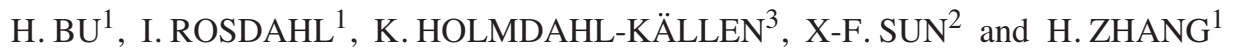 \\ Divisions of ${ }^{1}$ Dermatology and ${ }^{2}$ Oncology, Department of Biomedicine and Surgery, Linköping University, \\ SE-581 85 Linköping; ${ }^{3}$ Dermatology Clinic, Kalmar Hospital, SE-391 85 Kalmar, Sweden
}

Received November 9, 2006; Accepted December 14, 2006

\begin{abstract}
Polymorphisms of GSTM1, GSTT1 and GSTP1 were examined in melanoma patients and tumor-free individuals. Relationships between the polymorphisms and tumor characteristics and pigment phenotypes of the patients were analyzed. There was no significant difference in GSTM1 null and GSTT1 null genotypes nor GSTP1 GG genotype between melanoma patients and controls. In melanoma patients, these polymorphisms were not correlated with early or later onset of melanomas or gender of the patients. Frequency of GSTM1 null genotype was higher in patients with melanoma $>2.5 \mathrm{~mm}$ than in those with tumors $<1.0 \mathrm{~mm}$, and higher frequency was found in nodular melanoma than in the other tumor types. GSTP1 GG genotype was more often found in the patients with brown and mixed eye color or brown and black hair than those with blue and green eyes or blond hair. It is unlikely that polymorphisms of GSTM1, GSTT1 and GSTP1 are general risk factors for melanoma in the Swedish population. GSTM1 null genotype was correlated with Breslow thickness and tumor type, which might serve as an additional biomarker for a rapid tumor progression. GSTP1 GG increases risk for melanoma in the subgroup of individuals with dark eyes or hair.
\end{abstract}

\section{Introduction}

Incidence of melanoma has dramatically increased during the last decades. Although epidemiological studies show that ultraviolet (UV) radiation is a critical external risk factor for human skin melanoma the genetic predispositions for melanoma has not been fully elucidated (1-3).

A growing body of evidence demonstrates that DNA damage can be caused by direct UV radiation or indirect oxidative stress via reactive oxygen species induced from the

Correspondence to: Dr H. Zhang, Division of Dermatology, Department of Biomedicine and Surgery, Linköping University, SE-581 85 Linköping, Sweden

E-mail: hong.zhang@ibk.liu.se

Key words: GSTM1, GSTT1, GSTP1, polymorphisms, melanoma
UV radiation. If reactive oxygen species are not removed efficiently, they further result in DNA mutations and eventually tumorigenesis (4-6). Glutathione S-transferases (GSTs) are multifunctional enzymes involved in detoxification of a wide range of drugs and potential carcinogens, and are important players in the cellular defense systems $(7,8)$. The GSTs are the multigene superfamily consisting of 16 member genes divided into alpha (GSTA), mu (GSTM), omega (GSTO), pi (GSTP), theta (GSTT) and zeta (GSTZ). Among these genes, GSTT1, GSTM1 and GSTP1 commonly harbor functional polymorphisms in the general population (9). Homozygosity of null alleles in GSTM1 and GSTT1 genes result in gene deletion and deficiency of corresponding enzymes. Increase in frequencies of the null genotypes in GSTM1 and/or GSTT1 genes has been found to be associated with cancer susceptibility (10). Moreover, GSTP1 gene has an $\mathrm{A} \rightarrow \mathrm{G}$ polymorphism in its coding sequence, which results in an amino acid substitution from isoleucine to valine. The protein encoded by the $\mathrm{G}$ allele has a defective enzymatic activity as the residue lies in substrate-binding site and the substitution influences substrate-specific affinity and activity (11). GSTP1 homozygote GG genotype has been shown to be a predisposing risk factor in a number of human malignancies, such as bladder, leukemia and colorectal cancer (12-15).

In the present case-control study, we examined polymorphism genotypes of GSTM1, GSTT1 and GSTP1 genes in Swedish melanoma patients and age- and gender-matched tumor-free individuals as controls. Importance of these polymorphisms in melanoma risk was investigated. Further, associations of the genotypes with gender and age at tumor diagnosis, tumor-location, tumor-characteristics and pigment phenotype of the melanoma patients were analyzed.

\section{Patients and methods}

Melanoma patients and tumor-free population. The study was approved by the Regional Ethics Committee in Linköping University, Sweden. All patients were informed about the study details, and a written consent was obtained.

One hundred and fifty four patients (78 males and 76 females) with skin melanoma from southeastern region of Sweden participated in this study. The patients were diagnosed with primary melanoma in the University Hospital in Linköping and Kalmar Hospitals, Sweden. All patients were Caucasians with a mean age of 55.2 years (range 19-80 years). 


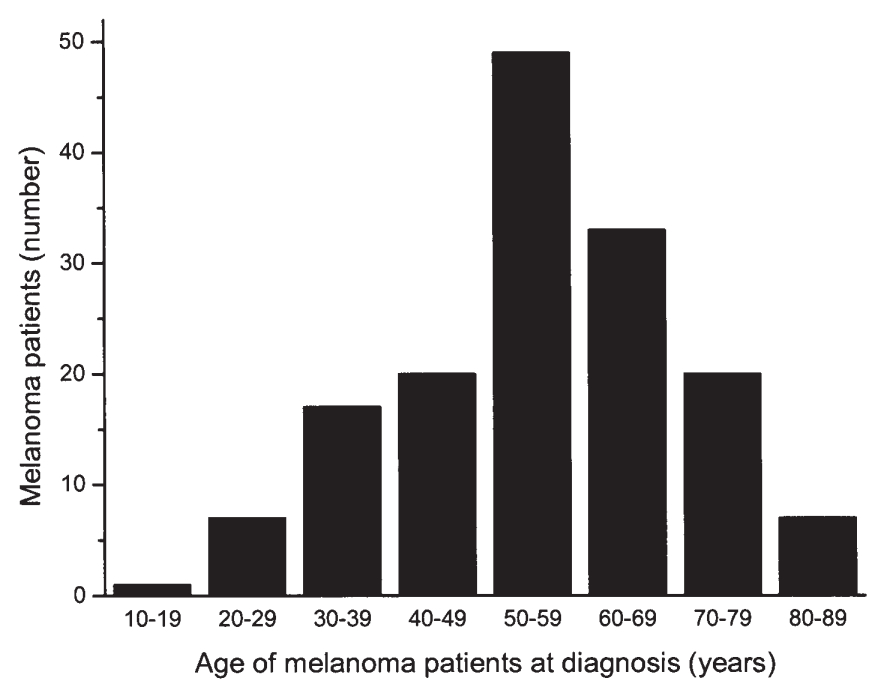

Figure 1. Age distribution at melanoma diagnosis $(n=154)$, mean age 55.2 years (range 19-80).

The age distribution at melanoma diagnosis is shown in Fig. 1. The control group from the same geographic region comprised 203 tumor-free healthy Caucasian individuals (100 males and 103 females). The mean age at the time of DNA collections was 55.2 years (range 22-77).

Tumor location was registered using a schematic body chart divided in 16 areas as described by Stierner et al (16). The areas were outlined taking clothing habits and general UV-exposure pattern into account which made it possible to classifying skin areas in those considered to be chronically, intermittently and rarely UV-exposed. Histopathology of tumor types, superficial spreading melanoma (SSM), nodular melanoma (NM), lentigo malignant melanoma (LMM) and acro-lentiginous melanoma (ALM) as well as Breslow thickness were reviewed from pathology records. Breslow thickness was classified in three groups $<1.0,1.0-2.5$ and $>2.5 \mathrm{~mm}$ (Table I).

Patient's pigment phenotype was registered by selfreporting. Skin type was categorized according to Melski et al (17). Analyses were performed between the two more sensitive skin types (I and II) and the two less sensitive ones (III and IV) grouped together. Eye color was graded in blue, green, brown and mixed, and hair color in blond, red, brown and black (Table II).

DNA isolation from mononuclear cells of venous blood. Venous blood $(5 \mathrm{ml})$ from each patient was collected in an anticoagulant tube and kept at $4^{\circ} \mathrm{C}$ less than a week for further DNA isolation. According to the manufacturer's instruction, genomic DNA was extracted from the mononuclear cells of venous blood by using DNA Blood Maxi Kit from Qiagen (Germany). DNA concentration from each sample was measured by an ND-1000 Spectrophotometer from Nanodrop (Wilmington, DE, USA). DNA working solution of $50 \mathrm{ng} / \mu \mathrm{l}$ in Milli-Q water was prepared from the original DNA stocks.

Genotyping of GSTM1, GSTT1 and GSTP1 genes by multiplex PCR and PCR-RFLP. Multiplex PCR was used to amplify fragments of GSTM1 and T1. In brief, three pairs of primers
Table I. Histopathological types and Breslow thickness of melanoma.

Variables

Melanoma patients no. $(\%)$

Histopathological type
In situ

Superficial spreading melanoma

Nodular melanoma

Lentigo malignant melanoma

Acro-lentiginous melanoma

Breslow thickness

$<1.0 \mathrm{~mm}$

$1.0-2.5 \mathrm{~mm}$

$>2.5 \mathrm{~mm}$
Table II. Pigment phenotypes of melanoma patients.

\begin{tabular}{lc}
\hline Phenotype & $\begin{array}{c}\text { Melanoma patients } \\
\text { no. }(\%)\end{array}$ \\
\hline Eye color & $130(84)$ \\
Blue + green & $24(16)$ \\
Brown + mixed & \\
Hair color & $129(84)$ \\
Blond + red & $25(16)$ \\
Brown + black & \\
Skin type & $80(52)$ \\
I+II & $74(48)$ \\
III+IV & \\
\hline
\end{tabular}

corresponding to GSTM1, GSTT1 and B-globin genes were added into the same PCR reaction system to detect the presence of GSTM1 or GSTT1. ß-globin gene was used as an internal positive control for successful amplification of PCR. The primers were purchased from Invitrogen (Scotland, UK). Primer sequences for GSTM1 were 5'-CGC CAT CTT GTG CTA CAT TGC CCG-3' and 5'-TTC TGG ATT GTA GCA GAT CA-3', producing a fragment of $231 \mathrm{bp}$, for GSTT1 were 5'-TTC CTT ACT GGT CCT CAC ATC TC-3' and 5'-TCA CCG GAT CAT GGC CAG CA-3', which produced a fragment of $460 \mathrm{bp}$, and for $\beta$-globin were 5'-CAA CTT CAT CCA CGT TCA CC-3' and 5'-GAA GAG CCA AGG ACA GGT AC-3', produced a fragment of $268 \mathrm{bp}$. The reaction was performed in $20 \mu \mathrm{l}$ mixture containing $50 \mathrm{ng}$ DNA, 1X PCR buffer from Promega (Madison, WI, USA), $0.5 \mu \mathrm{M}$ each primer, 0.5 U DNA polymerase (Promega), $1.875 \mathrm{mM} \mathrm{MgCl}_{2}$ (Promega), $0.25 \mathrm{mM}$ dNTP from Invitrogen (Carlsbad, CA, USA). The fragments were amplified with annealing temperature of $59^{\circ} \mathrm{C}$ for $90 \mathrm{sec}$ for 45 cycles. 


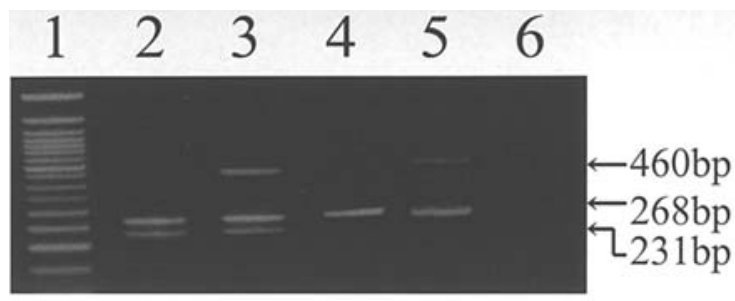

Figure 2. Genotypes of GSTM1 and GSTT1 in melanoma patients and tumorfree individuals were examined by multiplex PCR technique. The results were visualized in $3 \%$ agarose gel with $0.2 \mu \mathrm{g} / \mathrm{ml}$ ethidium bromide. Lane 1 , 100 bp DNA ladder; Lane 2, GSTM1 present and GSTT1 null genotype (231 bp); Lane 3, both GSTM1 and GSTT1 present genotype (460 and $231 \mathrm{bp}$ ); Lane 4, both GSTM1 and GSTT1 null genotype; Lane 5, GSTM1 null and GSTT1 present (460 bp) genotype; Lane 6, negative control. ß-globin fragment (268 bp) as internal control for DNA loading.

$\mathrm{A} \rightarrow \mathrm{G}$ polymorphism of GSTP1 was determined by PCRRFLP. Primers were 5'-ACC CCA GGG CTC TAT GGG AA-3' and 5'-TGA GGG CAC AAG AAG CCC CT-3', with a fragment $177 \mathrm{bp}$. In brief, the PCR was performed in $20 \mu \mathrm{l}$ mixture containing $50 \mathrm{ng}$ DNA, $1 \mathrm{X}$ PCR buffer, $1 \mu \mathrm{M}$ each primer, $2.25 \mathrm{mM} \mathrm{MgCl}_{2}, 0.5 \mathrm{U}$ DNA polymerase, $0.25 \mathrm{mM}$ dNTP. The fragment was amplified with annealing temperature of $62^{\circ} \mathrm{C}$ for $30 \mathrm{sec}$ for 40 cycles. The PCR product was digested with $10 \mathrm{U}$ of BsmAI in $1 \mathrm{X}$ buffer (New England Biolabs, UK) and incubated at $55^{\circ} \mathrm{C}$ for $18 \mathrm{~h}$. The $\mathrm{G}$ allele had a $B s m$ AI restriction site and was cleaved into two lengths of 84 and $93 \mathrm{bp}$, and the A allele was intact after BsmAI digestion. Both the multiplex PCR and restriction products were visualized in $3 \%$ agarose gel with $0.2 \mu \mathrm{g} / \mathrm{ml}$ ethidium bromide. DNA ladder (50 or $100 \mathrm{bp}$ ) was included in each run to determine the size of the fragments. PCR without DNA template was used as negative controls.

GSTM1 and GSTT1 individuals were divided into null or presence according to the absence or presence of the specific fragments. The group null was defined as subjects with homozygous deletion of GSTM1 or GSTT1, while presence included individuals with either homozygote or heterozygote of GSTM1 or GSTT1. GSTP1 genotype was distinguished as wild-type (AA), heterozygotes (AG) and polymorphic homozygotes (GG).

Statistical analysis. The $\chi^{2}$ test was used to estimate the differences in frequency of the genotypes and alleles between the melanoma patients and healthy controls, as well as within the subgroups of melanoma patients. All p-values shown are two-sided and $\mathrm{P}<0.05$ was judged as statistically significant. Risk of the genotypes was evaluated by odds ratio (OR) and 95\% confidence intervals $(\mathrm{CI})$.

\section{Results}

Polymorphisms of GSTM1, GSTT1 and GSTP1 and risk of melanoma. Genotypes of GSTM1, GSTT1 and GSTP1 were determined according to sizes of the PCR fragments in melanoma patients and tumor-free controls. As shown in Fig. 2, 231 bp fragments displayed the presence of GSTM1, $460 \mathrm{bp}$ fragments indicated the presence of GSTT1, and $268 \mathrm{bp}$ B-globin fragments revealed successful amplification of the

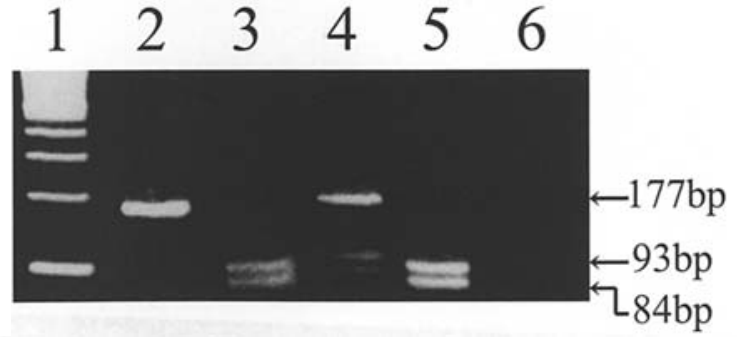

Figure 3. Genotypes of GSTP1 in melanoma patients and tumor-free individuals were examined by PCR-RFLP technique. The bands were visualized in $3 \%$ agarose gel with $0.2 \mu \mathrm{g} / \mathrm{ml}$ ethidium bromide. Lane $1,100 \mathrm{bp}$ DNA ladder; Lane 2, GSTP1 AA genotype (177 bp); Lanes 3 and 5, homozygous GSTP1 GG genotype (93 and 84 bp); Lane 4, GSTP1 heterozygous (AG) genotype (177,93 and 84 bp); Lane 6, negative control.

Table III. Distributions of GSTT1, GSTM1 and GSTP1 genotypes in 154 melanoma patients and healthy controls.

\begin{tabular}{lcccc}
\hline Genes & $\begin{array}{c}\text { Patients } \\
\text { no. }(\%)\end{array}$ & $\begin{array}{c}\text { Controls } \\
\text { no. }(\%)\end{array}$ & $\begin{array}{c}\text { OR } \\
(95 \% \text { CI })\end{array}$ & P-value \\
\hline GSTM1 & & & & \\
Presence & $81(52.6)$ & $99(48.8)$ & 1.0 & \\
Null & $73(47.4)$ & $104(51.2)$ & $0.86(0.56-1.30)$ & 0.47 \\
GSTT1 & & & & \\
Presence & $124(80.5)$ & $166(81.8)$ & 1.0 & \\
Null & $30(19.5)$ & $37(18.2)$ & $1.09(0.64-1.85)$ & 0.76 \\
GSTP1 & & & & \\
AA & $69(44.8)$ & $107(52.7)$ & 1.0 & \\
AG & $67(43.5)$ & $76(37.4)$ & $1.37(0.87-2.14)$ & \\
GG & $18(11.7)$ & $20(9.9)$ & $1.40(0.69-2.82)$ & 0.33 \\
\hline
\end{tabular}

samples. As shown in Fig. 3, GSTP1 wild-type AA genotype was demonstrated by the presence of a single $177 \mathrm{bp}$ fragment, and heterozygote AG genotype by the presence of 84, 93 and 177 bp fragments, and polymorphic homozygote GG genotype with the presence of 84 and 93 bp fragments.

There was no significant difference in the distribution of GSTM1 null (47.4 vs 51.2\%, P=0.47) and GSTT1 null (19.5 vs $18.2 \%, \mathrm{P}=0.76)$ genotypes, or in frequency of GSTP1 GG genotype (GG 11.7 vs $9.9 \%, \mathrm{P}=0.33$ ) between the melanoma patients and healthy controls (Table III).

Combinations of GSTM1, GSTT1 and GSTP1 genotypes were further evaluated for melanoma risk. Odds ratios for melanoma risk associated with various combinations of GST genotypes were calculated with GSTM1 and GSTT1 absence or presence, and GSTP1 AA group as reference. No significant difference in frequency of these genotype combinations was found between the melanoma patients and the tumor-free controls (Table IV).

Polymorphisms of GSTM1, GSTT1 and GSTP1 in melanoma patients. No associations between these polymorphisms and the gender or age at melanoma diagnosis (interval, 10 years) 
Table IV. Distribution of combinations of different GST genotypes in melanoma patients and healthy controls.

\begin{tabular}{lllrrr}
\hline GSTM1 & GSTT1 & GSTP1 & Patients $(\%)$ & Controls $(\%)$ & OR (95\% CI) \\
\hline Presence & Presence & AA & $29(18.8)$ & $41(20.2)$ & 1.0 \\
Presence & Presence & AG/GG & $36(23.4)$ & $42(20.7)$ & $1.21(0.63-2.32)$ \\
Presence & Null & AA & $4(2.6)$ & $10(4.9)$ & $0.57(0.16-1.98)$ \\
Null & Presence & AA & $27(17.5)$ & $45(22.2)$ & $0.85(0.43-1.66)$ \\
Presence & Null & AG/GG & $12(7.6)$ & $6(3.0)$ & $1.36(0.74-2.50)$ \\
Null & Presence & AG/GG & $32(20.8)$ & $38(18.7)$ & $1.19(0.61-2.32)$ \\
Null & Null & AA & $9(5.8)$ & $11(5.4)$ & $1.16(0.43-3.15)$ \\
Null & Null & AG/GG & $5(3.2)$ & $10(4.9)$ & $0.71(0.22-2.29)$ \\
\hline
\end{tabular}

Table V. Association of GSTM1 genotypes with Breslow thickness and histopathological type of melanoma.

\begin{tabular}{llll}
\hline & \multicolumn{2}{l}{ GSTM1 no. (\%) } \\
\cline { 2 - 3 } Variables & Null Presence & OR (95\% CI) & P-value
\end{tabular}

Breslow

thickness

$\begin{array}{lrrll}<1.0 \mathrm{~mm} & 25(39) & 39(61) & 1.0 & \\ 1.0-2.5 \mathrm{~mm} & 24(48) & 26(52) & 1.44(0.68-3.04) & \\ >2.5 \mathrm{~mm} & 11(73) & 4(27) & 4.29(1.23-14.94) & 0.03\end{array}$

Histopatho-

logical type

\begin{tabular}{lllll} 
SSM & $38(41)$ & $54(59)$ & 1.0 & \\
NM & $19(63)$ & $11(37)$ & $2.49(1.07-5.77)$ & 0.03 \\
\hline
\end{tabular}

was found. When Breslow thickness was analyzed an increased frequency of GSTM1 null genotype was found to be associated with increased tumor thickness $(39 \%$ in category $<1.0 \mathrm{~mm}$, $48 \%$ in category $1.0-2.5 \mathrm{~mm}$ and $73 \%$ in category $>2.5 \mathrm{~mm}$; Table V). The frequency of GSTM1 null genotype was significantly higher in melanoma patients with tumor thickness $>2.5 \mathrm{~mm}$, in comparison with the patients with tumor thickness $<2.5 \mathrm{~mm}(\mathrm{OR}=3.65,95 \% \mathrm{CI}=1.10-12.15, \mathrm{P}=0.03)$. Odds ratio was even higher when compared between patients with tumors $>2.5 \mathrm{~mm}$ and $<1.0 \mathrm{~mm}(\mathrm{OR}=4.29,95 \% \mathrm{CI}=$ 1.23-14.97, $\mathrm{P}=0.03)$. However, Breslow thickness was not associated with GSTT1 and GSTP1 polymorphisms.

Relationship of the polymorphism genotypes to histopathological types of melanoma was analyzed. Frequency of GSTM1 null genotype was significantly higher in the melanoma patients with NM than those with the other types of melanoma, including SSM, LMM and ALM (63 vs 41\%, $\mathrm{OR}=2.49,95 \% \mathrm{CI}=1.07-5.77, \mathrm{P}=0.03$; Table V). When frequency of the polymorphism in SSM (61\%) and NM (20\%) is compared we still see the significant different distribution between these two major types of melanomas. GSTT1 and GSTP1 polymorphisms were not found to be associated with the histopathological type of melanoma. Melanoma locations on the body analyzed in chronic, intermittent, or seldom sunexposed areas were not found to be associated with GSTM1, GSTT1 and GSTP1 genotypes in the patients.

Polymorphism of GSTP1 GG genotype and pigment phenotypes in melanoma patients. Interaction between GST polymorphisms and pigment phenotypes was evaluated in melanoma patients. The patients were classified according to their eye and hair color, and skin types. As shown in Table VI, we found a high frequency of GSTP1 GG genotype in melanoma patients with dark eyes compared to those with blue or green eyes ( 25 vs $10.9 \%, \mathrm{P}=0.027, \mathrm{OR}=3.28,95 \% \mathrm{CI}$ $=1.09-9.83)$. Moreover, patients with dark hair had a higher frequency of GSTP1 GG genotype compared to those patients with blond and red hair (28 vs $9.2 \%, \mathrm{P}=0.006$, $\mathrm{OR}=4.18$, $95 \% \mathrm{CI}=1.43-12.16)$. GSTP1 GG genotype was not found to be related to skin types ( $\mathrm{P}=0.24)$, and GSTT1 and GSTM1 polymorphisms were not associated with pigment phenotypes.

\section{Discussion}

Genetic predispositions and environment exposure such as UV radiation as risk factors for human skin melanoma (1-3). A sun-sensitive skin with a history of repeated and severe sunburns, especially during childhood, result in imbalance of the cellular oxidant-antioxidant system, leading to mutations and eventually to tumor growth (4-6). Moreover, variations in genes coding for different proteins involved in geneenvironmental interactions might be critical for melanoma induction as well as progression $(1,2)$. In the present study, we examined genomic polymorphisms of GSTM1, GSTT1 and GSTP1 in melanoma patients and tumor-free individuals. Associations of these polymorphisms with melanoma risk, patient's pigment phenotype and with tumor characteristics, especially tumor-progression related variables, such as Breslow thickness were analyzed. We did not find that frequencies of the GSTM1, GSTT1 polymorphisms in melanoma patients differ significantly from the tumor-free controls. Polymorphisms of these common functional genes in GSTs family are unlikely to play an important role in predisposition to human skin melanoma in the Swedish population. Heagerty et al have previously found that the frequency of GSTM1 null in 
Table VI. GSTP1 genotype and pigment phenotypes in melanoma patients.

\begin{tabular}{|c|c|c|c|c|}
\hline \multirow[b]{2}{*}{ Phenotypes } & \multicolumn{2}{|c|}{ GSTP1 genotypes } & \multirow[b]{2}{*}{ OR $(95 \%$ CI $)$} & \multirow[b]{2}{*}{ P-value } \\
\hline & GG (\%) & $\overline{\mathrm{AA}}+\mathrm{AG}(\%)$ & & \\
\hline \multicolumn{5}{|l|}{ Eye color } \\
\hline Blue + green & $12(10.9)$ & $118(89.1)$ & 1.0 & \\
\hline Brown + mixed & $6 \quad(25)$ & $18(75)$ & $3.28(1.09-9.83)$ & 0.03 \\
\hline \multicolumn{5}{|l|}{ Hair color } \\
\hline Blond + red & $11(9.2)$ & $118(90.8)$ & 1.0 & \\
\hline Brown + black & $7 \quad(28)$ & $18(72)$ & $4.18(1.43-12.16)$ & 0.006 \\
\hline \multicolumn{5}{|l|}{ Skin types } \\
\hline $\mathrm{I}+\mathrm{II}$ & $7 \quad(8.8)$ & 73 (91.2) & 1.0 & \\
\hline III+IV & $11(14.7)$ & $63(85.3)$ & $1.82(0.67-4.98)$ & 0.24 \\
\hline
\end{tabular}

cutaneous tumor cases and the control was similar in a population from the UK. However, the frequency of GSTM1 null was increased in multiple skin tumors of different types (18). In another report from a US population, GSTM1 and T1 was not found to be associated with susceptibility to skin melanoma in general, unlike the present results, however, they found that frequency of GSTM1 null was twice higher and both GSTM1 and T1 null was nearly 10-fold in the subgroup of patients with red or blond hair compared with the controls (19). The polymorphisms of the GST gene variants have been proven to be risk factors in lung cancer (20) and colorectal cancer (21). There are many other genetic predispositions, such as CDKN2A, BRAF, and NRAS which have been proven to play a role in susceptibility of human skin melanoma $(22,23)$.

In our study the GST polymorphisms did not correlate with patient's gender or age at melanoma diagnosis. We further analyzed the correlations between the GSTM1, GSTT1 and GSTP1 genotypes and clinicopathological characteristics of melanoma, and found that GSTM1 null genotype was independently associated with Breslow tumor thickness. The patients with thicker melanomas more often had GSTM1 null genotype than patients with thinner tumors. This might indicate that the GSTM1 is a genetic factor for tumor growth and progression. GSTM1 null individuals are deficient in reducing cellular oxidative stress in the melanocytes, which might result in a rapid tumor progression. Breslow thickness per se is one of the strongest prognostic markers in melanoma, and patients with thinner melanomas have a longer survival than those patients with thicker tumors (24). GSTM1 null genotype might together with other tumor prognostic parameters provide a stronger tumor growth indicator to predict survival for melanoma patients than Breslow thickness alone. This warrants further investigation, but has not been addressed in the present study. Frequency of GSTM1 null genotype was more common in the patients with NM tumors compared with the patients with other melanoma growth patterns (SSM, LMM and ALM). In general, NM progresses faster than the SSM type without or with a very short radical growth phase. GSTM1 null might provide a genetic marker for the rapid progression of this melanoma type although the exact mechanism behind this is unknown. Our result indicated that GSTM1 gene might be involved in formation of melanoma by driving the behavior of tumor cells toward different growth patterns.

We found that frequency of GSTP1 GG genotype was significantly higher in the patients with dark eyes or hair who generally have low risk for melanoma compared with those with blue eyes and blond hair. GSTP1 GG genotype might identify a subgroup of individuals with melanoma risk despite favorable pigment characteristics. The association with melanoma risk and GSTP1 GG genotype is however at present unexplained since the distribution of this genotype was similar in the group of patients with sun sensitivity and those with sun-tolerant skin.

This is the first study on polymorphisms of GSTM1, GSTT1 and GSTP1 genes in Swedish melanoma patients. In this study, we found that these polymorphisms did not play an important role as susceptible genes for identifying highrisk individuals. GSTP1 GG genotype in patients with dark eyes or darker hair is associated with an increased risk for melanoma in this subgroup of individuals.

The GSTM1 null genotype is a predictive marker for melanoma progression and predispose to a certain tumor growth pattern. GSTM1 null genotype might serve as an additional prognostic marker together with traditional markers. The GST polymorphisms are not associated with sun exposurerelated melanoma.

\section{Acknowledgements}

The authors are grateful for Mona-Lisa Sandh from the Department of Dermatology, Linköping University Hospital, Dr Kenneth Lagmo and Eva Niklasson from Dermatology Out-patient Clinic, Linköping, for kindly collecting blood samples from melanoma patients, and the melanoma patients who participate in this study. The study was supported by Cancer and Allergy Foundation, Edward Welander Foundation and Health Research Council in the Southeast of Sweden. 


\section{References}

1. Perlis $\mathrm{C}$ and Herlyn $\mathrm{M}$ : Recent advances in melanoma biology. Oncologist 9: 182-187, 2004.

2. Houghton AN and Viola MV: Solar radiation and malignant melanoma of the skin. J Am Acad Dermatol 5: 477-483, 1981.

3. Lejeune FJ: Epidemiology and etiology of malignant melanoma. Biomed Phamacother 40: 91-99, 1986.

4. Douki T, Reynaud-Angelin A, Cadet J and Sage E: Bipyrimidine photoproducts rather than oxidative lesions are the main type of DNA damage involved in the genotoxic effect of solar UVA radiation. Biochemistry 42: 9221-9226, 2003.

5. Rochette PJ, Therrien JP, Drouin R, et al: UVA-induced cyclobutane pyrimidine dimers form predominantly at thyminethymine dipyrimidines and correlate with the mutation spectrum in rodent cells. Nucleic Acids Res 31: 2786-2794, 2003.

6. Dahle J and Kvam E: Induction of delayed mutations and chromosomal instability in fibroblasts after UVA-, UVB-, and X-radiation. Cancer Res 63: 1464-1469, 2003.

7. Lusini L, Tripodi SA, Rossi R, et al: Altered glutathine antioxidant metabolism during tumor progression in human renalcell carcinoma. Int J Cancer 91: 55-59, 2001.

8. Baars AJ and Breimer DD: The glutathione S-transferases: their role in detoxification and toxification of xenobiotics. Ann Biol Clin 38: 49-56, 1980.

9. Nebert DW and Vasiliou V: Analysis of the glutathione S-transferase (GST) gene family. Hum Genomics 1: 460-464, 2004.

10. Rebbeck TR: Molecular epidemiology of the human glutathione S-transferase genotypes GSTM1 and GSTT1 in cancer susceptibility. Cancer Epidemiol Biomarkers Prev 6: 733-774, 1997.

11. Henderson CJ, McLaren AW, Moffat GJ, Bacon EJ and Wolf CR: $\mathrm{Pi}$-class glutathione S-transferase: regulation and function. Chem Biol Intertact 111-112: 69-82, 1998.

12. Srivastava DS, Mishra DK, Mandhani A, Mitta B, Kumar A and Mittal RD: Association of genetic polymorphism of glutathione S-transferase M1, T1, P1 and susceptibility to bladder cancer. Eur Urol 48: 339-344, 2005.

13. Ye Z and Song H: Glutathione S-transferase polymorphisms (GSTM1, GSTP1 and GSTT1) and the risk of acute leukaemia: a systematic review and meta-analysis. Eur J Cancer 41: 980-989, 2005.
14. Sun X-F, Ahmadi A, Arbman G, et al: Polymorphisms in sulfotransferase $1 \mathrm{~A} 1$ and glutathione S-transferase P1 genes in relation to colorectal cancer risk and patients' survival. World J Gastroenterol 11: 6875-6879, 2005.

15. Zhang H, Ahmadi A, Arbman G, et al: Gluthathine S-transferase T1 and M1 genotypes in normal mucosa, transitional mucosa and colorectal adenocarcinoma. Int J Cancer 84: 135-138, 1999.

16. Stierner U, Augustsson A, Rosdahl I and Suurkula M: Regional distribution of common and dysplastic naevi in relation to melanoma site and sun exposure. A case-control study. Melanoma Res 1: 367-375, 1992.

17. Melski JW, Tannenbaum L, Parrish JA, Fitzpatrick TB and Bleich HL: Oral methoxalen photochemotherapy for the treatment of psoriasis: a cooperative clinical trial. J Invest Dermatol 68: 328-335, 1977.

18. Heagerty AH, Fitzgerald D, Smith A, et al: Glutathione S-transferase GSTM1 phenotypes and protection against cutaneous tumours. Lancet 343: 266-268, 1994.

19. Kanetsky PA, Homes R, Walker A, et al: Interaction of glutathione S-transferase M1 and T1 genotypes and malignant melanoma. Cancer Epidemiol Biomarkers Prev 10: 509-513, 2001.

20. Ye Z, Song H, Higgins J, Pharoah P and Danesh J: Five glutathione S-transferase gene variants in 23,452 cases of lung cancer and 30,397 controls: meta-analysis of 130 studies. PLoS Med 3: 1-11, 2006.

21. Welfare M, Monesola Adeokun A, Bassendine MF, Bassendine MF and Daly AK: Polymorphisms in GSTP1, GSTM1 and GSTT1 and susceptibility to colorectal cancer. Cancer Epidemiol Biomarkers Prev 8: 289-292, 1999.

22. De Soon FA and Hayward NK: Cutaneous melanoma susceptibility and progression genes. Cancer Lett 230: 153-186, 2005.

23. Curtin JA, Fridlyand J, Kageshita T, et al: Distinct sets of genetic alterations in melanoma. N Engl J Med 353: 2135-2147, 2005 .

24. Mackie RM, Smyth JF, Soutar DS, et al: Malignant melanoma in Scotland 1979-1983. Lancet 2: 859-863, 1985. 